\title{
Numerical simulation of posture in 3-DOF parallel mechanism based on VB
}

\author{
GuoXuzhuo $^{1, a}$, Shi Yanbin ${ }^{1, b}$, Liu Jingang ${ }^{1}$, Fu Tinggui ${ }^{1}$, Pan Chunxiang ${ }^{1}$ \\ ${ }^{1}$ Aviation University of Air Force, Changchun 130022,China \\ ${ }^{2}$ Department of Designing, HUAWEI Co., Ltd., Shenzhen 518000,China \\ aguoxuzhuo@163.com, bshiyanbin_80@163.com
}

\begin{abstract}
Keywords: Parallel mechanism; Posture model; Numerical simulation
Abstract. In this paper, a forward displacement solution of 3 degree of freedom (3-DOF) parallel mechanism is primarily resolved. The close loop equation is adopted for the positional posture analysis of the output displacement of spatial 3-RPS parallel mechanism, the forward displacement of the mechanism is educed, and the positional posture of output displacement is acquired. Finally, numerical examples are performed for the positional posture analysis of the mechanism.
\end{abstract}

\section{Introduction}

3-DOF parallel mechanism has been widely used in the independent mechanism, joint mechanism、 machine tool worktable, positioning workpiece and so on[1] because of their valuable advantages which embrace high stiffness, high precision、 simple structure, easy manufacturing and convenient access to other mechanisms. Inverse displacement solution of parallel mechanism is easier to get while forward displacement solution is extremely complicated, therefore the focus on forward displacement solution is far less than inverse displacement solution. As for forward solution, there are mainly two ways to acquire, one is numerical method and the other is analytical method ${ }^{[1]}$. It is easy to get mathematical model and suit most parallel mechanisms in numerical method but not all the solutions will be acquired. All the solutions will be acquired in analytical method and the error between import and export will be expressed quantitatively but it is hard to deduce ${ }^{[2-4]}$.

In this paper, the method of getting forward displacement solution of 3-DOF parallel mechanism has been primarily discussed. The positional posture of the output piece of spatial 3-RPS parallel mechanism is analyzed by use of close loop equation to get the kinematics forward solution, which means the positional posture of output piece is confirmed. Finally, numerical simulation examples are performed for the positional posture analysis of the mechanism.

\section{Description of mechanism}

Structural parameters of spatial 3-RPS parallel mechanism ${ }^{[5]}$ are shown in Fig.1. This parallel mechanism is composed of base platform, mobile platform and three branches. Mobile platform and branches are connected by spherical pairs, base platform and branches are connected by rotational pairs, and translational pairs on the branches serve as driving pairs. Changing length of the three branches will inevitably lead to the changes of position and posture of mobile platform.

Coordinate system of spatial 3-RPS parallel mechanism are shown in Fig.2. Base platform is triangle $A_{1} A_{2} A_{3}$, mobile platform is triangle $B_{1} B_{2} B_{3}$, and three branches are $A_{1} B_{1} 、 A_{2} B_{2}$ and $A_{3} B_{3}$ and $A_{i}\left(x_{A i}\right.$ , $\left.\mathrm{y}_{\mathrm{Ai}}, 0\right)$ and $\mathrm{B}_{\mathrm{i}}\left(\mathrm{x}_{\mathrm{Bi}}, \mathrm{y}_{\mathrm{Bi}}, \mathrm{z}_{\mathrm{Bi}}\right)(\mathrm{i}=1,2,3)$ respectively represent the center of rotational pairs and spherical pairs. Base coordinate system $A_{1}$-xyz is set up, origin lies in the position $A_{1}$, axis $x$ goes along $\mathrm{A}_{1} \mathrm{~A}_{2}$, axis $\mathrm{z}$ goes upwards and is vertical to base platform, and axis $\mathrm{z}$ is confirmed by the right hand rule. $\stackrel{i}{i}, \dot{j} 、 k^{v}$ respectively stand for direction unit vectors of the three axes. Side length of the base 
platform is expressed by $a_{i}$, and its direction unit vector is expressed by $\stackrel{\mathrm{v}}{a}_{i}$; Side length of the mobile platform is expressed by $b_{i}$, and its direction unit vector is expressed by $b_{i}$; length of three branches is expressed by $p_{i}$, and its direction unit vector is expressed by $\stackrel{v}{p_{i}}$.

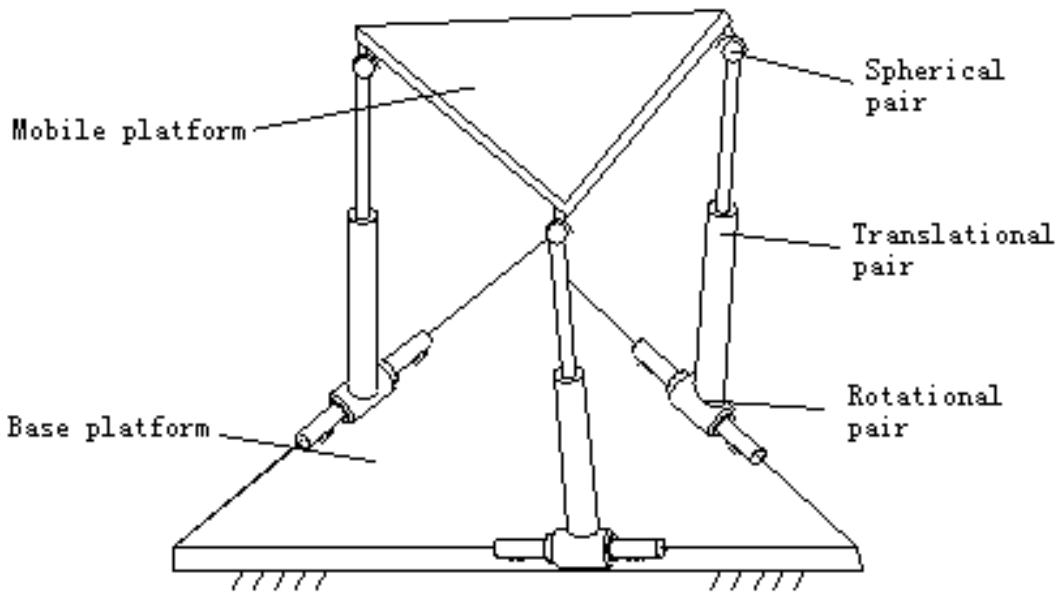

Fig.1. Sketch of 3-RPS mechanism

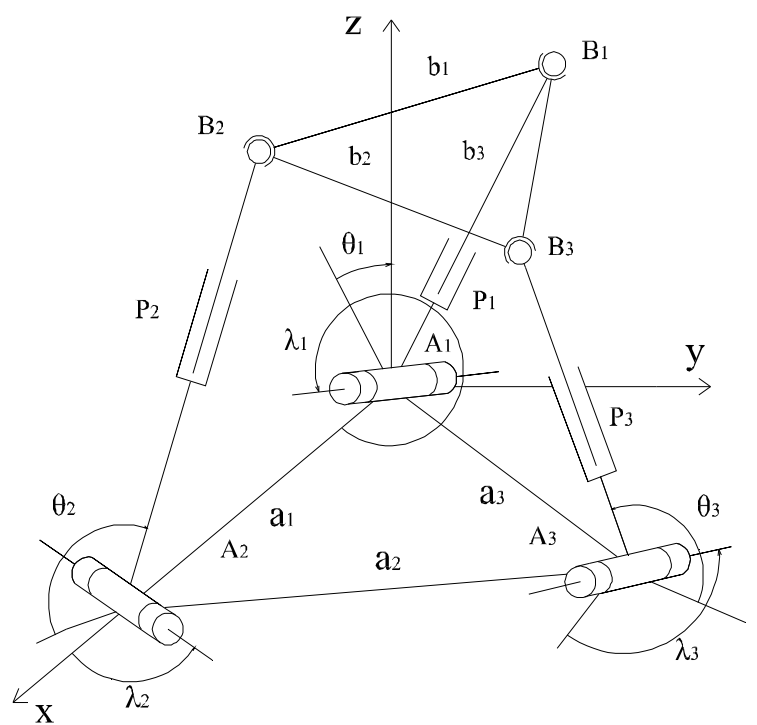

Fig.2. Coordinate system of 3-RPS mechanism

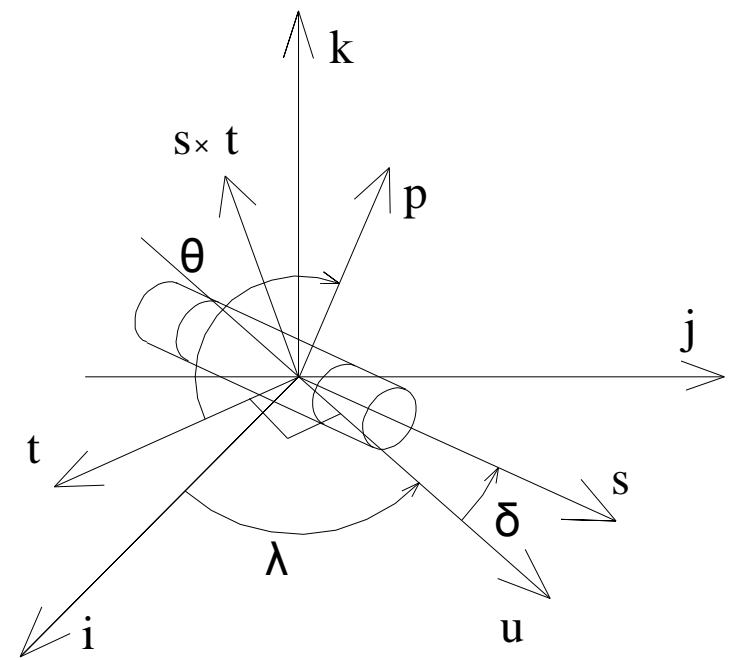

Fig.3. Direction unit vector of parallel mechanism

The connections among all direction unit vectors have been expressed in Fig.3. Direction unit vector along rotational pair axis is $s_{i}$, and $s_{i}$ is projected on the plane of $\mathrm{xA}_{1} \mathrm{y}$, then $\underset{v}{u_{i}}$ is the direction unit vector of projection. $\lambda_{i}$ is angle between $\breve{u}_{i}$ and $\stackrel{v}{i}, \delta_{i}$ is angle between $\breve{u}_{i}$ and $\stackrel{v}{s}_{i}, \breve{v}_{i}$ is direction unit vector which is vertical to both rotational pair axis and $\mathrm{z}$ axis. $\theta_{i}$ is rotational angle among three branches, and $\alpha_{i}$ is angle between base platform sides and positive $\mathrm{x}$ axis. Therefore, the equations are following.

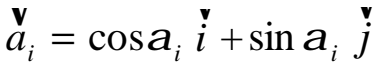

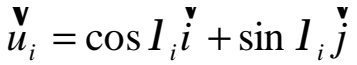

$$
\begin{aligned}
& \stackrel{\mathrm{v}}{t_{i}}=\sin \lambda_{i} \stackrel{\mathrm{v}}{i}-\cos \lambda_{i} \stackrel{\mathrm{v}}{j} \\
& \stackrel{r}{s_{i}}=\cos \lambda_{i} \cos \delta_{i} \stackrel{\dot{i}}{+}+\sin \lambda_{i} \cos \delta_{i}{ }^{\prime} \dot{j}+\sin \delta_{i} \dot{k}
\end{aligned}
$$




$$
\begin{aligned}
& {\stackrel{\mathrm{v}}{p_{i}}}=\cos \theta_{i} \stackrel{\mathrm{t}}{\mathrm{t}}_{i}+\sin \theta_{i}\left(\check{t}_{i} \times{\stackrel{\mathrm{v}}{s_{i}}}\right) \\
& =\cos \theta_{i}\left(\sin \lambda_{i} \stackrel{\mathrm{v}}{i}-\cos \lambda_{i} \stackrel{\stackrel{v}{j}}{j}\right)+\sin \theta_{i}\left(-\cos \lambda_{i} \sin \delta_{i} \stackrel{\mathrm{v}}{i}-\sin \delta_{i} \sin \lambda_{i} \stackrel{\mathrm{v}}{j}+\cos \delta_{i} \ddot{k}^{\mathrm{v}}\right)
\end{aligned}
$$

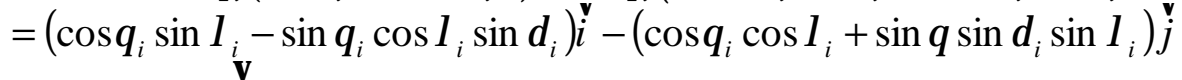

$$
\begin{aligned}
& +\cos \delta_{i} \sin \theta_{i} k
\end{aligned}
$$

\section{Numerical simulation}

Numerical simulation has been done based on Visual Basic ${ }^{[6-7]}$ to check out the actual pose of mechanism. Where the parameters of mechanism are $a_{1}=a_{2}=a_{3}=600(\mathrm{~mm})$ 、 $b_{1}=b_{2}=b_{3}=300(\mathrm{~mm}) 、 \alpha_{1}=0^{\circ}, \alpha_{2}=120^{\circ} 、 \alpha_{3}=240^{\circ} 、 \lambda_{1}=300^{\circ} 、 \lambda_{2}=60^{\circ} 、 \lambda_{3}=180^{\circ}$. According to equations from (14) to (16), input variable $p_{1} 、 p_{2}$ are taken as a fixed value, and $p_{3}$ continuously changes within a certain range, then curves will be obtained( $p_{3}-\theta_{1}$ curve 、 $p_{3}-\theta_{2}$ curve and $p_{3}-\theta_{3}$ curve)

Example 1 Input displacement $p_{1}=377, p_{2}=477, p_{3}=370 \sim 820(\mathrm{~mm})$

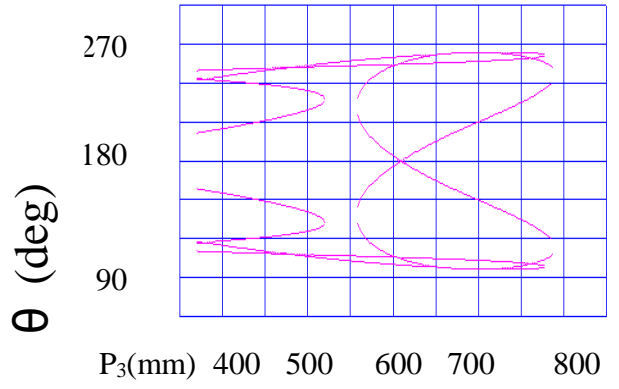

Fig. $3.1 p_{3}-\theta_{1}$ curve
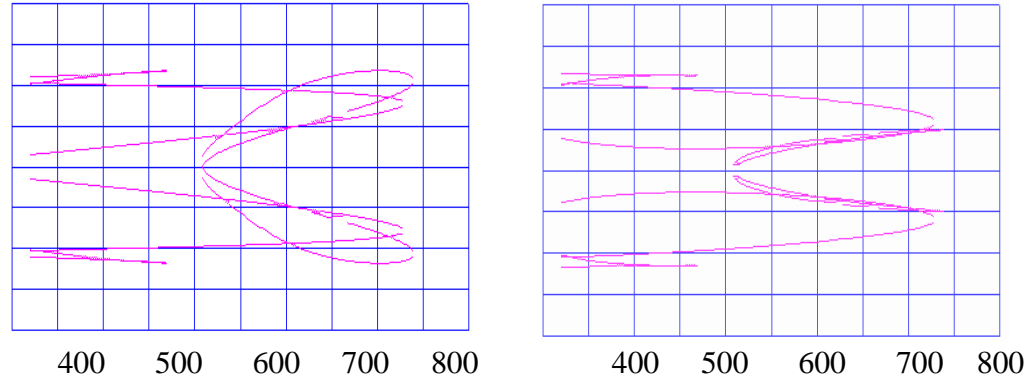

Fig. $3.2 p_{3}-\theta_{2}$ curve

Fig. $3.3 p_{3}-\theta_{3}$ curve

Example 2 Input displacement $p_{1}=377, p_{2}=527, p_{3}=370 \sim 820(\mathrm{~mm})$

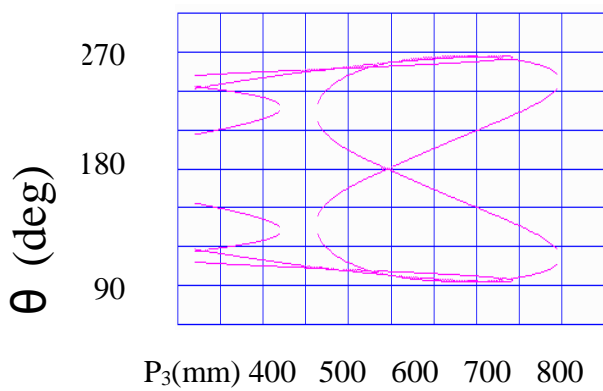

Fig. $3.4 p_{3}-\theta_{1}$ curve

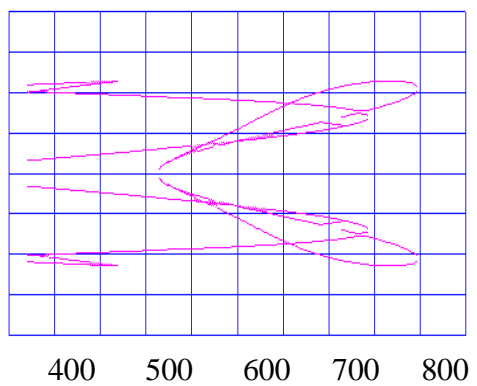

Fig. $3.5 p_{3}-\theta_{2}$ curve

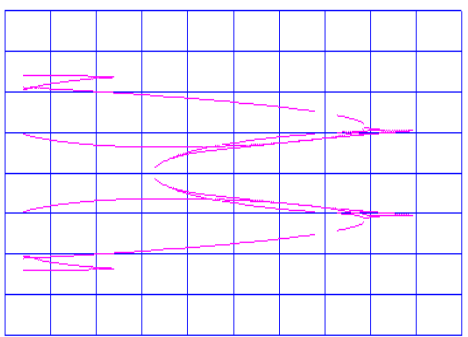

$\begin{array}{lllll}400 & 500 & 600 & 700 & 800\end{array}$

Fig. $3.6 p_{3}-\theta_{3}$ curve

Seen from Fig.3.1 to Fig3.6, Input displacement $p_{1} 、 p_{2}$ are fixed values, $p_{3}-\theta_{1}$ curve 、 $p_{3}-\theta_{2}$ curve and $p_{3}-\theta_{3}$ curve will be changing followed by the changes of variable $p_{3}$. All these curves are symmetrical about horizontal line because of geometrical symmetry of mechanism. Where there is discontinuity of curves, there is limiting position of mechanism, and the motion will be absent of regularity, therefore the limiting position should be especially considered when analyzing properties of mechanism.

\section{Conclusion}

3-RPS spatial three-DOF parallel mechanism is analyzed in this paper. Kinematic forward solution is solved by use of closed loop equations, connections between output variables and input variables are 
expressed by curves, and the regularity of pose are also be obtained, which is useful for further analysis of mechanism properties ${ }^{[8-9]}$ and also meaningful for other form of parallel mechanism.

\section{Acknowledgements}

This work was financially supported by Education Department of Jilin Province "13th Five-Year" scientific research planning project(2016-514).

\section{References}

[1] Huang Zhen , Kong lingfu. Mechanism theory and control of parallel robot.BeijingMachinery industry press, 1997.

[2] Zhang Xiufeng, Sun Lining. Technical analysis and prospect of parallel robot.High technology communications. .2004,6:107 110 .

[3] Chen Xuesheng, Chen Zaili. Progress and current situation of parallel robot.Robot, 2002, 24(5): $464 \sim 470$.

[4] Song Xin. Application of welding swing mechanism in 3-RPS parallel platform.Welding, 2004,4:24 26.

[5] Fang Yuefa, Huang Zhen. Instant independent motion of 3-RPS parallel robot' $\mathrm{s}$ manipulator. Mechanical science and technology. 1996,15(6):929 934.

[6] Fang Yuefa, Huang Zhen. Motional analysis of 3-RPS parallel robot.Mechanical science and technology. 1997,16(1):82 88.

[7] Yang Ping. Pose analysis of hydraulic turbine repair robot.Mechanical design. 2004,21(10) : 11 $\sim 13$.

[8] Lu Yue,WangPeng,Hou zhuolei.Static analysis of a novel 6 DOF parallel manipulator with multi-fingers. Mechanism and machine theory. 2014,8(78): $36 \sim 50$.

[9] MengXiang,GaoFeng,WuShengfu.TYpe Synthesis of parallel robotic mechanms :Frame work and brief review. Mechanism and machine theory. 2014,8(78): $117 \sim 186$. 